\title{
CYLINDER FUNCTIONS IN THE FRESNEL CLASS OF FUNCTIONS ON ABSTRACT WIENER SPACES
}

\author{
DONG MYUNG CHUNG AND HONG TAEK HWANG
}

(Communicated by Andrew M. Bruckner)

\begin{abstract}
In this paper we consider the class of cylinder functions on abstract Wiener space $B$ and give necessary and sufficient conditions of cylinder functions on $B$ to be in the Banach algebra $\mathfrak{F}(B)$ (resp. $\mathfrak{F}^{*}(B)$ ) of analytic (resp. sequential) Feynman integrable functions on $B$. The results here subsume similar known results obtained by Chang, Johnson, and Skoug in the setting of Hilbert and Wiener spaces.
\end{abstract}

\section{INTRODUCTION AND PRELIMINARIES}

Let $(H, B, \nu)$ be an abstract Wiener space where $H$ is a separable Hilbert space with the inner product $\langle\cdot, \cdot\rangle$ and the norm $|\cdot|=\sqrt{\langle\cdot, \cdot\rangle}$, which is densely and continuously embedded into a separable Banach space $B$ with the norm $\|\cdot\|$, and $\nu$ is the abstract Wiener measure on the Borel $\sigma$-algebra $\mathfrak{B}(B)$ of $B$ that is generated by the Gauss cylinder set measure on $H$ with mean zero, variance one. As $H$ is identified as a dense subspace of $B$, we identified the topological dual $B^{*}$ of $B$ as a dense subspace of $H^{*}=H$ in the sense that for all $y$ in $B^{*}$ and $x$ in $H,\langle y, x\rangle=(y, x)$ where $(\cdot, \cdot)$ is the $B^{*}-B$ pairing. Thus we have a triple $B^{*} \subset H \subset B$. For more details, see [16]. Let $\mathbb{R}^{n}$ and $\mathbb{C}$ denote an $n$-dimensional Euclidean space and the complex numbers, respectively.

Let $\left\{e_{j} ; j \geq 1\right\}$ be a complete orthonormal set in $H$ such that $e_{j}$ 's are in $B^{*}$. For each $h \in H$ and $x \in B$, we define

$$
(h, x)^{\sim}=\lim _{n \rightarrow \infty} \sum_{j=1}^{n}\left\langle h, e_{j}\right\rangle\left(e_{j}, x\right)
$$

if the limit exists and $=0$ otherwise. It is well known that for each $(h \neq 0)$ in $H,(h, \cdot)^{\sim}$ is a Gaussian random variable on $B$ with mean zero, variance $|h|^{2}$.

Let $M(H)$ be the class of all $\mathbb{C}$-valued Borel measures on $H$. Then $M(H)$ is a Banach algebra under the total variation norm where convolution is taken

Received by the editors September 18, 1990.

1991 Mathematics Subject Classification. Primary 46G12, 28 C20.

The first author's research was partially supported by BSRIP, MOE, Korea.

(C) 1992 American Mathematical Society $0002-9939 / 92 \$ 1.00+\$ .25$ per page 
as the multiplication. The Fourier transform of $\mu$ in $M(\mu)$ is defined for all $h_{1}$ in $H$ by

$$
\hat{\mu}\left(h_{1}\right)=\int_{H} \exp \left\{i\left\langle h, h_{1}\right\rangle\right\} d \mu(h) .
$$

The Fresnel class $\mathfrak{F}(H)$ of functions on $H$ is defined [1, p. 17] as the space of all Fourier transforms of elements of $M(H)$.

Given two $\mathbb{C}$-valued measurable functions $F$ and $G$ on $B, F$ is said to be equal to $G s$-almost surely (s-a.s.) if for each $\alpha>0, \nu\{x \in B: F(\alpha x) \neq$ $G(\alpha x)\}=0$ (for more detail, see $[8,11]$ ). We write that $F \approx G$ (resp. $F \approx G$ ) if $F=G s$-a.s. (resp. if $F \approx G$ and furthermore if $F(x)=G(x)$ for all $x \in H)$. Both relations $\approx$ and $\approx$ are clearly equivalence relations. We use the notation $[F]$ for the equivalence class of $F$ with respect to the relation $\approx$. We also use the same notation $[F]$ for the relation $\approx$. by

The Fourier transform $\hat{\mu}$ of the form (1.1) can be extended to $B$ uniquely

$$
\hat{\mu}^{\sim}(x)=\int_{H} \exp \left\{i(h, x)^{\sim}\right\} d \mu(h) .
$$

We shall consider the following two Fresnel classes of functions on $B$ given by

$$
\mathfrak{F}(B)=\left\{[F]: F(x) \approx \hat{\mu}^{\sim}(x), \mu \in M(H)\right\}
$$

and

$$
\mathfrak{F}^{*}(B)=\left\{[F]: F(x) \approx \hat{\mu}^{\sim}(x), \mu \in M(H)\right\} .
$$

As is customary, we think of the elements of $\mathfrak{F}(B)\left(\mathfrak{F}^{*}(B)\right)$ as functions on $B$ rather than equivalence classes. It was shown in $[14,15]$ that $\mathfrak{F}(B)$ and $\mathfrak{F}^{*}(B)$ are Banach algebras with norm $\|F\|=\|\hat{\mu}\|$ and that the elements of $\mathfrak{F}(B)$ (resp. $\mathfrak{F}^{*}(B)$ ) are analytic (resp. sequential) Feynman integrable.

A function $F$ is a cylinder function on $B$ if there exist $\varphi_{1}, \varphi_{2}, \ldots, \varphi_{k} \in H$ such that

$$
F(x)=f\left(\left(\varphi_{1}, x\right)^{\sim},\left(\varphi_{2}, x\right)^{\sim}, \ldots,\left(\varphi_{k}, x\right)^{\sim}\right),
$$

where $f$ is a Borel measurable function on $\mathbb{R}^{k}$. It can be shown that there exists a linearly independent subset $\left\{h_{1}, h_{2}, \ldots, h_{n}\right\}$ of $H$ such that $F$, with the form (1.2), is written as

$$
F(x)=\psi\left(\left(h_{1}, x\right)^{\sim},\left(h_{2}, x\right)^{\sim}, \ldots,\left(h_{n}, x\right)^{\sim}\right),
$$

where $\psi$ is a Borel measurable function on $\mathbb{R}^{n}$. Thus we lose no generality in assuming that every cylinder function on $B$ is of the form (1.3).

Let $h(\neq 0) \in H$ and $F: B \rightarrow \mathbb{C}$ be a cylinder function on $B$ defined by $F(x)=\psi\left((h, x)^{\sim}\right)$ where $\psi$ is in $L^{1}\left(\mathbb{R}^{1}\right)$ but not in $L^{\infty}\left(\mathbb{R}^{1}\right)$. Then it is easy to see that $F$ is not in $\mathfrak{F}(B)$. Thus we see that every cylinder function on $B$ is not necessarily in $\mathfrak{F}(B)$. Motivated by this fact and the works of Chang, Johnson, and Skoug [4-7], we consider the class of cylinder functions on abstract Wiener space $B$ and give necessary and sufficient conditions for the cylinder functions on $B$ to be in a Banach algebra $\mathfrak{F}(B)$ (resp. $\mathfrak{F}^{*}(B)$ ) of analytic (resp. sequential) Feynman integrable functions on $B$. The results here subsume similar known results given in $[4,5]$ by Chang, Johnson, and Skoug. We finally apply our results to a classical Wiener space $C[0, t]$ to obtain results 
in $[4,5]$ as corollaries and obtain various functions on $C[0, t]$ that are Feynman integrable (cf. $[6,7])$.

\section{NECESSARY AND SUFFICIENT CONDITIONS FOR CYLINDER FUNCTIONS ON $B$ TO BE IN $\mathfrak{F}(B)$}

In this section we begin with two necessary lemmas to prove the main results of this paper.

Lemma 2.1. Let $(H, B, \nu)$ be an abstract Wiener space. Let $\left\{h_{1}, h_{2}, \ldots, h_{n}\right\}$ be a linearly independent subset of $H$ and $\lambda: B \rightarrow \mathbb{R}^{n}$ be defined by

$$
\lambda(x)=\left(\left(h_{1}, x\right)^{\sim},\left(h_{2}, x\right)^{\sim}, \ldots,\left(h_{n}, x\right)^{\sim}\right) .
$$

Let $\psi$ and $\eta$ be $\mathbb{C}$-valued Borel measurable functions on $\mathbb{R}^{n}$. Then

(i) $\psi=\eta$ a.e. on $\mathbb{R}^{n}$ if and only if $\psi \circ \lambda \approx \eta \circ \lambda$.

(ii) $\psi=\eta$ everywhere on $\mathbb{R}^{n}$ if and only if $\psi \circ \lambda \approx \eta \circ \lambda$.

Proof. (i) Let $J=\{x \in B: \psi(\lambda(x)) \neq \eta(\lambda(x))\}$ and $D=\left\{\vec{r} \in \mathbb{R}^{n}: \psi(\vec{r}) \neq\right.$ $\eta(\vec{r})\}$. Then we have $J=\{x \in B: \lambda(x) \in D\}$. We note that $\rho J=\lambda^{-1}(\rho D)$ for all $\rho>0$. Since $\lambda$ is a nondegenerate Gaussian random vector, we see that $\nu \circ \lambda^{-1}$ is equivalent to Lebesgue measure on $\mathbb{R}^{n}$. Hence for all $\rho>0$, $\nu(\rho J)=\nu \circ \lambda^{-1}(\rho D)=0$ if and only if $\rho D$ is null in $\mathbb{R}^{n}$. Therefore $\psi=\eta$ a.e. on $\mathbb{R}^{n}$ if and only if $\psi \circ \lambda \approx \eta \circ \lambda$.

(ii) Since $\lambda(H)=\mathbb{R}^{n}$, we have that $\psi=\eta$ on $\mathbb{R}^{n}$ if and only if $\psi \circ \lambda(x)=$ $\eta \circ \lambda(x)$ for all $x \in H$. Further, we have that $\psi \circ \lambda=\eta \circ \lambda$ on $H$ if and only if $\psi(\lambda(x)) \approx \eta(\lambda(x))$ for all $x \in B$. Hence we complete the proof.

Lemma 2.2. Let $\left\{h_{1}, h_{2}, \ldots, h_{n}\right\}$ be a linearly independent subset of $H$. Let $\mu \in M(H)$ be such that

$$
\hat{\mu}^{\sim}(x+p) \approx \hat{\mu}^{\sim}(x) \text { for every } p \in\left[h_{1}, h_{2}, \ldots, h_{n}\right]^{\perp},
$$

where $[A]^{\perp}$ is the orthogonal complement of the subspace of $H$ spanned by $A$. Then there exists a measure $\sigma \in M\left(\mathbb{R}^{n}\right)$ such that

$$
\hat{\mu}^{\sim}(x)=\hat{\sigma}\left(\left(h_{1}, x\right)^{\sim},\left(h_{2}, x\right)^{\sim}, \ldots,\left(h_{n}, x\right)^{\sim}\right) .
$$

Proof. Let $\rho \in \mathbb{R}^{1}$ and $p \in\left[h_{1}, \ldots, h_{n}\right]^{\perp}$ be arbitrary but fixed. Then by the hypothesis, $\hat{\mu}^{\sim}(x+\rho p) \approx \hat{\mu}(x)$. Thus we have

$$
\int_{H} \exp \left\{i(h, x)^{\sim}\right\} d \mu(h) \approx \int_{H} \exp \left\{i(h, x)^{\sim}\right\} d \mu_{\rho}(h),
$$

where $d \mu_{\rho}(h)=\exp \{i \rho\langle h, p\rangle\} d \mu(h)$. Hence we have that $\hat{\mu}^{\sim} \approx \hat{\mu}_{\rho}^{\sim}$. By Proposition 2.1 in [14], it follows that $\mu=\mu_{\rho}$. By using a theorem in [17, p. 133], there exists a $\mathbb{C}$-valued measurable function $g$ with its absolute value 1 such that $d \mu(h)=g(h) d|\mu|(h)$. Thus $d \mu=d \mu_{\rho}$ can be written as

$$
g(h) d|\mu|(h)=\exp \{i \rho\langle h, p\rangle\} g(h) d|\mu|(h) .
$$

By the Radon-Nykodym theorem it follows that $\exp \{i \rho\langle h, p\rangle\}=1$ for $|\mu|$-a.e. $h$. Since $\rho$ was an arbitrary real number, we must have $\langle h, p\rangle=0$ for $|\mu|$-a.e. 
$h$. Further, since $p$ was also an arbitrary element in $\left[h_{1}, h_{2}, \ldots, h_{n}\right]^{\perp}$, we have that

$$
h \in\left[h_{1}, h_{2}, \ldots, h_{n}\right]^{\perp \perp}=\left[h_{1}, h_{2}, \ldots, h_{n}\right] \text { for }|\mu| \text {-a.e. } h .
$$

Therefore the measure $\mu$ is supported by $\left[h_{1}, h_{2}, \ldots, h_{n}\right]$.

Now let $\varphi:\left[h_{1}, h_{2}, \ldots, h_{n}\right] \rightarrow \mathbb{R}^{n}$ be the homeomorphism defined by $\varphi(h)=$ $\left(\left\langle h, h_{1}\right\rangle, \ldots,\left\langle h, h_{n}\right\rangle\right)$. Then the measure $\sigma \equiv \mu \circ \varphi^{-1}$ is in $M\left(\mathbb{R}^{n}\right)$. Since $\mu$ is supported by $\left[h_{1}, \ldots, h_{n}\right]$, we have

$$
\begin{aligned}
\hat{\mu}^{\sim}(x) & =\int_{H} \exp \left\{i(h, x)^{\sim}\right\} d \mu(h)=\int_{\left[h_{1}, \ldots, h_{n}\right]} \exp \left\{i(h, x)^{\sim}\right\} d \mu(h) \\
& =\int_{\left[h_{1}, \ldots, h_{n}\right]} \exp \left\{i\left(\sum_{j=1}^{n}\left\langle h, h_{j}\right\rangle h_{j}, x\right)^{\sim}\right\} d \mu(h) \\
& =\int_{\left[h_{1}, \ldots, h_{n}\right]} \exp \{i\langle\varphi(h), \lambda(x)\rangle\} d \mu(h) \\
& =\int_{\mathbb{R}^{n}} \exp \{i\langle y, \lambda(x)\rangle\} d \mu \circ \varphi^{-1}(y)=\hat{\sigma}(\lambda(x)),
\end{aligned}
$$

where $\lambda(x)$ is as in $(2.1)$.

Theorem 2.3. Let $\left\{h_{1}, h_{2}, \ldots, h_{n}\right\}$ be a linearly independent subset of $H$. Let $F: B \rightarrow \mathbb{C}$ be a cylinder function on $B$ as in (1.3). Then

(a) $F$ is in $\mathfrak{F}(B)$ if and only if there exists a measure $\sigma \in M\left(\mathbb{R}^{n}\right)$ such that $\hat{\sigma}=\psi$ a.e. on $\mathbb{R}^{n}$.

(b) $F$ is in $\mathfrak{F}^{*}(B)$ if and only if there exists a measure $\sigma \in M\left(\mathbb{R}^{n}\right)$ such that $\hat{\sigma}=\psi$ everywhere on $\mathbb{R}^{n}$.

Proof. (a) Let $F \in \mathfrak{F}(B)$. Then there exists a measure $\mu \in M(H)$ such that $\hat{\mu}^{\sim}(x) \approx F(x)$. Since $\left(h_{j}, x+p\right)^{\sim}=\left(h_{j}, x\right)^{\sim}$ for all $j=1, \ldots, n$ and for all $p \in\left[h_{1}, \ldots, h_{n}\right]^{\perp}$, we clearly have that $\hat{\mu}^{\sim}(x+p) \approx \hat{\mu}^{\sim}(x)$ for all $p \in\left[h_{1}, \ldots, h_{n}\right]^{\perp}$. Hence by Lemma 2.2, there exists a measure $\sigma \in M\left(\mathbb{R}^{n}\right)$ such that

Thus we have

$$
\hat{\mu}^{\sim}(x)=\hat{\sigma}\left(\left(h_{1}, x\right)^{\sim}, \ldots,\left(h_{n}, x\right)^{\sim}\right), \quad x \in B .
$$

$$
\psi\left(\left(h_{1}, x\right)^{\sim}, \ldots,\left(h_{n}, x\right)^{\sim}\right) \approx \hat{\sigma}\left(\left(h_{1}, x\right)^{\sim}, \ldots,\left(h_{n}, x\right)^{\sim}\right) .
$$

Hence by Lemma 2.1(i), we have that $\psi=\hat{\sigma}$ a.e. on $\mathbb{R}^{n}$.

Conversely, let $\sigma \in M\left(\mathbb{R}^{n}\right)$ be such that $\psi=\hat{\sigma}$ a.e. on $\mathbb{R}^{n}$. Then by Lemma 2.1(i), we have

$$
\psi\left(\left(h_{1}, x\right)^{\sim}, \ldots,\left(h_{n}, x\right)^{\sim}\right) \approx \hat{\sigma}\left(\left(h_{1}, x\right)^{\sim}, \ldots,\left(h_{n}, x\right)^{\sim}\right) .
$$

Define $\phi: \mathbb{R}^{n} \rightarrow H$ by $\phi\left(\left(r_{1}, r_{2}, \ldots, r_{n}\right)\right)=\sum_{j=1}^{n} r_{j} h_{j}$. Set $\mu=\sigma \circ \phi^{-1}$. Then $\mu$ is in $M(H)$ and is supported by $\left[h_{1}, h_{2}, \ldots, h_{n}\right]$. Hence we have

$$
\begin{aligned}
\hat{\mu}^{\sim}(x) & =\int_{H} \exp \left\{i(h, x)^{\sim}\right\} d \mu(h)=\int_{\left[h_{1}, \ldots, h_{n}\right]} \exp \left\{i(h, x)^{\sim}\right\} d \sigma \circ \phi^{-1}(h) \\
& =\int_{\mathbb{R}^{n}} \exp \left\{i(\phi(\vec{r}), x)^{\sim}\right\} d \sigma(\vec{r})=\hat{\sigma}\left(\left(h_{1}, x\right)^{\sim}, \ldots,\left(h_{n}, x\right)^{\sim}\right)
\end{aligned}
$$

from which $F$ is in $\mathfrak{F}(B)$. 
(b) Let $F \in \mathfrak{F}^{*}(B)$. Then there exists a $\mu \in M(H)$ such that

$$
\hat{\mu}^{\sim}(x) \approx \psi\left(\left(h_{1}, x\right)^{\sim}, \ldots,\left(h_{n}, x\right)^{\sim}\right) .
$$

So $\hat{\mu}(h)=\psi\left(\left\langle h_{1}, h\right\rangle, \ldots,\left\langle h_{n}, h\right\rangle\right)$ for all $h \in H$. Using Theorem 8 of [4], we have a $\sigma \in M\left(\mathbb{R}^{n}\right)$ such that $\psi=\hat{\sigma}$ on $\mathbb{R}^{n}$.

Conversely, let $\psi=\hat{\sigma}$ on $\mathbb{R}^{n}$ with $\sigma \in M\left(\mathbb{R}^{n}\right)$. Then by Lemma 2.1(ii), $\psi\left(\left(h_{1}, x\right)^{\sim}, \ldots,\left(h_{n}, x\right)^{\sim}\right) \approx \hat{\sigma}\left(\left(h_{1}, x\right)^{\sim}, \ldots,\left(h_{n}, x\right)^{\sim}\right)$. Let $\mu=\sigma \circ \phi^{-1}$ where $\phi$ is as in the proof of (a). Then $\mu \in M(H)$ and

$$
\hat{\mu}^{\sim}(x)=\hat{\sigma}\left(\left(h_{1}, x\right)^{\sim}, \ldots,\left(h_{n}, x\right)^{\sim}\right)
$$

from which $F$ is in $\mathfrak{F}^{*}(B)$.

Remark 2.1. An alternative proof of (b) can be given as follows: Let $f=\left.F\right|_{H}$. Then $f \in \mathfrak{F}(H)$ if and only if $F \in \mathfrak{F}^{*}(B)$ (see [15]). But by Theorem 8 of [4], $f \in \mathfrak{F}(H)$ if and only if $\psi=\hat{\sigma}$ on $\mathbb{R}^{n}$ where $\sigma \in M\left(\mathbb{R}^{n}\right)$. Hence the proof of (b) follows.

\section{Corollaries}

In this section we apply our results in the preceding section to classical Wiener space to obtain results in $[4,5]$ as corollaries and obtain further corollaries.

For simplicity, we consider the case $(H, B, \nu)=\left(C^{\prime}[0, t], C[0, t], m_{w}\right)$, where $C[0, t]$ is the Wiener space, $m_{w}$ is the Wiener measure, and $C^{\prime}[0, t]=$ $\left\{x \in C[0, t]: x(s)=\int_{0}^{s} f(u) d u, f \in L^{2}[0, t]\right\}$ is a real separable infinitedimensional Hilbert space with inner product $\left\langle x_{1}, x_{2}\right\rangle=\int_{0}^{t} D x_{1}(\tau) \cdot D x_{2}(\tau) d \tau$, where $D x=d x / d \tau$ (see [16]).

We first note (see $[14,16])$ that if $B=C[0, t]$ and $H=C^{\prime}[0, t]$, then for $h \in H$ and $x \in B$,

$$
(h, x)^{\sim}=\int_{0}^{t} D h(s) \tilde{d} x(s),
$$

where $\int_{0}^{t} D h(s) \tilde{d} x(s)$ is the Paley-Wiener-Zygmund integral of $D h$.

In $[2,3]$ Cameron and Storvick introduced Banach algebras $S$ and $S^{*}$ of functions on $C[0, t]$ given by

$$
S=\left\{F: F(x) \approx \int_{L^{2}[0, t]} \exp \left\{i \int_{0}^{t} v(s) \tilde{d} x(s)\right\} d \mu(v), \mu \in M\left(L^{2}[0, t]\right)\right\}
$$

and

$$
\begin{aligned}
S^{*}=\{F: F(x) & \approx \int_{L^{2}[0, t]} \exp \left\{i \int_{0}^{t} v(s) \tilde{d} x(s)\right\} d \mu(v), \mu \in M\left(L^{2}[0, t]\right), \\
F(x) & \left.=\int_{L^{2}[0, t]} \exp \left\{i \int_{0}^{t} v(s) \tilde{d} x(s)\right\} d \mu(v) \text { for all } x \in C^{\prime}[0, t]\right\},
\end{aligned}
$$

and then showed $[2,3]$ that the analytic (resp. sequential) Feynman integral exists for all elements of $S$ (resp. $S^{*}$ ). 
Remark 3.1. (1) Let $I$ be the unitary operator from $L^{2}(\mathbb{R})$ onto $C^{\prime}[0, t]$ given by $I v(\tau)=\int_{0}^{\tau} v(s) d s$. If

$$
F(x) \approx \int_{L^{2}[0, t]} \exp \left\{i \int_{0}^{t} v(s) \tilde{d} x(s)\right\} d \mu(v)
$$

for some $\mu \in M\left(L^{2}[0, t]\right)$, then we have

$$
F(x) \approx \int_{C^{\prime}[0, t]} \exp \left\{i(h, x)^{\sim}\right\} d \mu \circ I^{-1}(h) .
$$

Conversely, if

$$
F(x) \approx \int_{C^{\prime}[0, t]} \exp \left\{i(h, x)^{\sim}\right\} d \eta(h)
$$

for some $\eta \in M\left(C^{\prime}[0, t]\right)$, then we have

$$
F(x) \approx \int_{L^{2}[0, t]} \exp \left\{i \int_{0}^{t} v(s) \tilde{d} x(s)\right\} d \eta \circ I(v) .
$$

Thus we have that $F \in S$ if and only if $F \in \mathfrak{F}(C[0, t])$ (see [14]). Similarly, we have that $F \in S^{*}$ if and only if $F \in \mathfrak{F}^{*}(C[0, t])$.

(2) Let $A: C^{\prime}[0, t] \rightarrow C^{\prime}[0, t]$ be the linear operator defined by

$$
(A g)(\tau)=\int_{0}^{\tau} g(u) d u
$$

Then we see that the adjoint operator $A^{*}$ of $A$ is given by

$$
\left(A^{*} g\right)(\tau)=g(t) \tau-\int_{0}^{\tau} g(u) d u \text {. }
$$

It is easily shown that $A^{*}$ is injective.

Throughout the rest of this section we assume that $\psi$ is a $\mathbb{C}$-valued Borel measurable function on $\mathbb{R}^{n}$.

Corollary 3.1. Let $F_{1}: C[0, t] \rightarrow \mathbb{C}$ be given by

$$
F_{1}(x)=\psi\left(\int_{0}^{t} v_{1}(s) x(s) d s, \ldots, \int_{0}^{t} v_{n}(s) x(s) d s\right),
$$

where $\left\{v_{1}, \ldots, v_{n}\right\}$ is a linearly independent subset of $L^{2}[0, t]$. Then

(1) $F_{1} \in S$ if and only if $\psi=\hat{\sigma}$ a.e. on $\mathbb{R}^{n}$ where $\sigma \in M\left(\mathbb{R}^{n}\right)$.

(2) $F_{1} \in S^{*}$ if and only if $\psi=\hat{\sigma}$ on $\mathbb{R}^{n}$ where $\sigma \in M\left(\mathbb{R}^{n}\right)$.

Proof. Let $h_{j}(\tau)=\int_{0}^{\tau} v_{j}(s) d s$ for $j=1,2, \ldots, n$. Then $h_{j} \in C^{\prime}[0, t]$ and $\left\{h_{1}, h_{2}, \ldots, h_{n}\right\}$ is a linearly independent subset of $C^{\prime}[0, t]$. Let $k_{j}(\tau)=$ $\left(A^{*} h_{j}\right)(\tau)$ for $j=1,2, \ldots, n$, where $A^{*}$ is as in (3.2). Since $A^{*}$ is linear and injective, $\left\{k_{1}, k_{2}, \ldots, k_{n}\right\}$ is an independent subset of $C^{\prime}[0, t]$. Further we note that

$$
\left(A^{*} h_{j}, x\right)^{\sim}=\int_{0}^{t} D\left(A^{*} h_{j}\right)(s) \tilde{d} x(s)=\int_{0}^{t} x(s) v_{j}(s) d s .
$$

Hence $F_{1}(x)=\psi\left(\left(A^{*} h_{1}, x\right)^{\sim}, \ldots,\left(A^{*} h_{n}, x\right)^{\sim}\right)$ is a cylinder function on $C[0, t]$. Therefore the proof follows by Theorem 2.3. 
Corollary 3.2. Let $F_{2}: C[0, t] \rightarrow \mathbb{C}$ be given by

$$
F_{2}(x)=\psi\left(\int_{0}^{s_{1}} x(s) d s, \ldots, \int_{0}^{s_{n}} x(s) d s\right),
$$

where $0<s_{1}<s_{2}<\cdots<s_{n} \leq t$. Then the conclusions (1) and (2) of Corollary 3.1 are true for $F_{2}$.

Proof. Apply Corollary 3.1 with $v_{j}(s)=1_{\left[0, s_{j}\right]}(s)$ for $j=1,2, \ldots, n$.

Corollary 3.3. Let $F_{3}: C[0, t] \rightarrow \mathbb{C}$ be given by

$$
F_{3}(x)=\psi\left(\frac{1}{s_{1}} \int_{0}^{s_{1}} x(s) d s, \frac{1}{s_{1}-s_{2}} \int_{s_{1}}^{s_{2}} x(s) d s, \ldots, \frac{1}{s_{n}-s_{n-1}} \int_{s_{n-1}}^{s_{n}} x(s) d s\right),
$$

where $0<s_{1}<s_{2}<\cdots<s_{n} \leq t$. Then the conclusions (1) and (2) of Corollary 3.1 are true for $F_{3}$.

Proof. Apply Corollary 3.1 with

$$
v_{j}(s)=\frac{1}{s_{j}-s_{j-1}} 1_{\left[s_{j-1} s_{j}\right]}(s)
$$

for $j=1,2, \ldots, n$, where $s_{0}=0$.

Remark 3.2. Let $\eta$ be a finite Borel measure on $[0, t]^{n}$. Let $\theta:[0, t]^{n} \times \mathbb{R}^{n} \rightarrow \mathbb{C}$ be given by

$$
\theta(\vec{r}, \vec{u})=\int_{\mathbb{R}^{n}} \exp \{i\langle\vec{u}, \vec{w}\rangle\} d \sigma_{\vec{r}}(\vec{w}),
$$

where $\left\{\sigma_{\vec{r}}, \vec{r} \in[0, t]^{n}\right\}$ is a family from $M\left(\mathbb{R}^{n}\right)$ such that for every $B \in \mathfrak{B}\left(\mathbb{R}^{n}\right)$ $\sigma_{\vec{r}}(B)$ is Borel measurable in $\vec{r}$, and where $\left\|\sigma_{\vec{r}}\right\| \in L^{1}\left([0, t]^{n}, \eta\right)$. Then by Theorem 1 of [7], we have that the functions on $C[0, t]$ given by

$$
F_{4}(x)=\int_{[0, t]^{n}} \theta\left(\vec{s}, \int_{0}^{s_{1}} x(s) d s, \ldots, \int_{0}^{s_{n}} x(s) d s\right) d \eta(\vec{s})
$$

and

$$
F_{5}(x)=\int_{[0, t]^{n}} \theta\left(\vec{s}, \int_{0}^{s_{1}} x(s) d s, \ldots, \int_{s_{n-1}}^{s_{n}} x(s) d s\right) d \eta(\vec{s})
$$

are both in $S$. Furthermore, $\exp \left\{F_{4}(x)\right\}$ and $\exp \left\{F_{5}(x)\right\}$ are also both in $S$.

Corollary 3.4. Let $F_{6}: C[0, t] \rightarrow \mathbb{C}$ be given by

$$
F_{6}(x)=\psi\left(\int_{0}^{t} v_{1}(s) \tilde{d} x(s), \ldots, \int_{0}^{t} v_{n}(s) \tilde{d} x(s)\right),
$$

where $\left\{v_{1}, \ldots, v_{n}\right\}$ is a linearly independent subset of $L^{2}[0, t]$. Then the conclusions (1) and (2) of Corollary 3.1 are true for $F_{6}$.

Proof. Let $I$ be as in Remark 3.1. Then we have

$$
F_{6}(x)=\psi\left(\left(I v_{1}, x\right)^{\sim}, \ldots,\left(I v_{n}, x\right)^{\sim}\right) .
$$

So by Theorem 2.3 it follows that $F_{6} \in \mathfrak{F}(C[0, t])$ (resp. $\mathfrak{F}^{*}(C[0, t])$ ) if and only if there exist a $\sigma \in M\left(\mathbb{R}^{n}\right)$ such that $\psi=\hat{\sigma}$ a.e. (resp. everywhere) on $\mathbb{R}^{n}$. Hence from Remark 3.1 we complete the proof. 
Corollary 3.5. Let $0<s_{1}<s_{2}<\cdots<s_{n}=t$ be a partition of $[0, t]$ and let

$$
F_{7}(x)=\psi\left(x\left(s_{1}\right), x\left(s_{2}\right)-x\left(s_{1}\right), \ldots, x\left(s_{n}\right)-x\left(s_{n-1}\right)\right) .
$$

Then the conclusions (1) and (2) of Corollary 3.1 are true for $F_{7}$.

Proof. Apply Corollary 3.4 with $v_{j}(s)=1_{\left[s_{j-1}, s_{j}\right]}(s)$ for $j=1,2, \ldots, n$, where $s_{0}=0$.

Corollary 3.6. Let $0<s_{1}<s_{2}<\cdots<s_{n}=t$ be a partition of $[0, t]$ and let

$$
F_{8}(x)=\psi\left(x\left(s_{1}\right), x\left(s_{2}\right), \ldots, x\left(s_{n}\right)\right) .
$$

Then the conclusions (1) and (2) of Corollary 3.1 are true for $F_{8}$.

Proof. Apply Corollary 3.4 with $v_{j}(s)=1_{\left[0, s_{j}\right]}(s)$ for $j=1,2, \ldots, n$.

\section{REFERENCES}

1. S. Albeverio and R. Hoegh-Krohn, Mathematical theory of Feynman path integrals, Lecture Notes in Math., vol. 523, Springer-Verlag, Berlin, 1976.

2. R. H. Cameron and D. A. Storvick, Some Banach algebras of analytic Feynman integrable functionals in analytic functions, Kozubnik, 1979, Lecture Notes in Math., vol. 798, Springer-Verlag, Berlin, 1980, pp. 18-67.

3. $\ldots$ A simple definition of the Feynman integral with applications, Mem. Amer. Math. Soc., vol. 46, no. 288, Amer. Math. Soc., Providence, RI, 1983, pp. 1-46.

4. K. S. Chang, G. W. Johnson, and D. L. Skoug, Necessary and sufficient conditions for the Fresnel integrability of certain classes of functions, J. Korean Math. Soc. 21 (1984), 21-29.

5. __ Necessary and sufficient conditions for membership in the Banach algebra $S$ for certain classes of functions, Circ. Mat. Palermo. (suppl.) 17 (1987), 153-171.

6. __ Functions in the Fresnel class, Proc. Amer. Math. Soc. 100 (1987), 309-318.

7. _, Functions in the Banach algebra $S(\nu)$, J. Korean Math. Soc. 24 (1987), 121-132.

8. Dong M. Chung, Scale-invariant measurability in abstract Wiener spaces, Pacific J. Math. 130 (1987), 27-40.

9. Dong M. Chung and Soon J. Kang, Evaluation formulas for conditional abstract Wiener integrals, Stochastic Anal. Appl. 7 (1989), 125-144.

10. G. W. Johnson, The equivalence of two approaches to the Feynman integral, J. Math. Phys. 23 (1982), 2090-2096.

11. G. W. Johnson and D. L. Skoug, Scale-invariant measurability in Wiener space, Pacific J. Math. 83 (1979), 157-176.

12. _ _ Notes on the Feynman integral. I, Pacific J. Math. 93 (1981), 313-324.

13. _ Notes on the Feynman integral III: The Schroedinger equation, Pacific J. Math. 105 (1983), 321-358.

14. G. Kallianpur and D. C. Bromley, Generalized Feynman integrals using analytic condition in several complex variables, Stochastic Analysis and Applications (M. A. Pinsky, ed.), Marcel-Dekker, New York, 1984.

15. G. Kallianpur, D. Kannan, and R. L. Karandikar, Analytic and sequential Feynman integrals on abstract Wiener and Hilbert spaces and a Cameron-Martin formula, Annales de l'Institut Henri Poincaré-Probabilités et Statistiques, 21 (1985), 323-361.

16. H. H. Kuo, Gaussian measure in Banach spaces, Lecture Notes in Math., vol. 463, SpringerVerlag, Berlin, 1975.

17. W. Rudin, Real and complex analysis, MacGraw-Hill, New York, 1966.

Department of Mathematics, Sogang University, Seoul 121-742, Korea KOREA

Department of Applied Mathematics, Kum-oh Institute of Technology, Kumi 730-701, 